\title{
Higher phenolic acid excretion in polypectomy patients at risk of colorectal cancer
}

\author{
A. Alkhaldy ${ }^{1}$, C. A. Edwards ${ }^{1}$, C. Roxburgh ${ }^{2}$ and E. Combet ${ }^{1}$ \\ ${ }^{1}$ Human Nutrition, School of Medicine, College of MVLS, University of Glasgow, Glasgow, G3 8SJ, UK and \\ ${ }^{2}$ University Department of Surgery, University of Glasgow, Royal Infirmary, Glasgow, G4 OSF, UK
}

Plant foods contain a range of bioactive molecules, including polyphenols, which may impact on the gut microbiota and on the risk of colorectal cancer $(\mathrm{CRC})^{(1)}$. The bioavailability and metabolism of polyphenols have been studied in young adults, and their colonic metabolism to phenolic acids may be key for their effects on colonic cells. Little is known about the impact of ageing on polyphenol metabolism and how this impacts on gut health of older adults and those at risk of $\mathrm{CRC}^{(2)}$. The colonic microbiota changes with ageing along with, an increase in inflammatory status, which may affect gut health and colorectal cancer risk ${ }^{(3)}$. These age-related changes may also influence the colonic metabolism of polyphenols and their impact on gut health. In this pilot study, we hypothesized that people at risk of CRC ( $>50$ years old and a history of adenoma removal) may metabolise dietary polyphenols less efficiently than healthy counterparts.

Healthy participants (aged $54-75 ; n=15)$ and patients at risk of CRC (51-63y; $n=7$, polypectomy in the previous $2-8$ weeks) followed a 3-day low polyphenol diet and 3-day high-polyphenol diet consecutively. Weighed dietary records and a food frequency questionnaire (FFQ) focusing on polyphenol-rich foods were completed. Urine and faeces were collected at the end of each diet. Urinary phenolic acids ( $n=17$; benzoic acid, phenylacetic acid, mandelic acid, 3-hydroxybenzoic acid, 3-hydroxyphenylacetic acid, 4-hydroxybenzoic acid, 4-hydroxyphenylpropionic acid, vanillic acid, homovanillic acid, 4-hydroxymandelic acid, 3,4-dihydroxybenzoic acid, 3,4-dihydroxyphenylacetic acid, hippuric acid, dihydrocaffeic acid, 4-hydroxy-3-methoxy-phenylpropionic acid, gallic acid, and 4-hydroxyhippuric acid) were measured by GC-MS. Faecal short chain fatty acids (SCFA) were measured by GC-FID as an index of colonic bacterial fermentation. Faecal pH was also measured. Bifidobaceria and Bacteroidetes (modulated by polyphenolics) and Flavonifractor plautii (a known flavonoid degrader) were measured by real-time quantitative PCR. Results were compared with Wilcoxon sign rank test, and Mann-Witney U-test as appropriate.

Urinary phenolic acids excretion significantly increased after the high-polyphenol diet $(p=0.0003)$ from 68.0 (SD 66.3 ) to 423.1 (SD 431.9) $\mu \mathrm{g} /$ day in the healthy group and $(p=0.02)$ from 233.21 (SD 143.8) to 788.1 (SD 438.3) $\mu \mathrm{g} /$ day in the CRC risk group. The difference in excretion ( $\Delta$ low-high diet) was not different between groups, however, phenolic acid concentration was higher in the urine of $\mathrm{CRC}$ risk volunteers after both diets, than in healthy older volunteers $(p<0.05)$. There were no significant changes in SCFA concentration after the high polyphenol diet in either group (from 226.4 (SD 69.2) to 254.7 (SD 86.4 ) $\mu$ moles/g in the healthy group, and 321.4 (SD 166.9) to 261.8 (SD 51.8) $\mu$ moles/g in the CRC risk group). No significant difference ( $\Delta$ low-high diet) was observed between groups. Faecal $\mathrm{pH}$ decreased $(p<0.05)$ in both groups, with no significant difference between groups. Faecal concentration of Bifidobacteria was similar in healthy and CRC risk groups $(9.4 \log 10$ copies/g wet wt (SD 0.7$)$ versus 8.9 log10 copies/g wet wt (SD 0.3), respectively). Similarly, there were no differences in Bacteroidetes $(10.0 \log 10$ copies/g wet wt (sD 0.3) versus $9.6 \log 10$ copies/g wet wt (SD 0.5), respectively), or Flavonifractor plautii (7.8 $\log 10$ copies/g wet wt (SD 0.6) versus 8.0 log10 copies/g wet wt (SD 0.6), respectively. The difference seen in phenolic acid excretion could not be accounted for by changes in specific bacteria groups or species but the sample may be too small to detect this.

The reasons and consequences of the higher urinary phenolic acid excretion after polypectomy needs to be explored in a larger study designed utilising the data from this pilot.

This work was supported by the Faculty of Applied Medical Sciences - Clinical Nutrition Department, King Abdulaziz University, Jeddah, Kingdom of Saudi Arabia 2010-1013, and Tenovus Scotland.

1. Bosscher D, Breynaert A, Pieters L et al. (2009) J of phys \& pharm 60, 5-11.

2. Borges G, Mullen W, Mullan A et al. (2010) Mol Nutr Food Res 54, 268-277.

3. Garagnani P, Pirazzini C \& Franceschi C (2013) Curr pharm des 19(4), 765-78. 